\title{
The Effect of Transdermal Glyceryl Trinitrate in Acute Stroke Patients with Carotid Stenosis: Data from the Efficacy of Nitric Oxide in Stroke
}

Jason P Appleton, Lisa J Woodhouse, Zhe Kang Law, Nikola Sprigg, Philip M Bath for the ENOS Investigators

E NOS

Stroke Trials Unit, Division of Clinical Neuroscience, University of Nottingham, Nottingham, UK

University of Nottingham UK | CHINA | MALAYSIA

\section{BACKGROUND}

There is concern that blood pressure lowering in acute stroke may compromise cerebral perfusion and worsen outcome in the context of carotid stenosis.

The effect of glyceryl trinitrate (GTN) on outcome in acute stroke patients with carotid stenosis is unclear.

We sought to assess GTN's effect in this context using data from the Efficacy of Nitric Oxide in Stroke (ENOS) trial.

\section{METHODS}

ENOS randomised 4011 patients with acute stroke ( $<48$ hours) and raised systolic BP (SBP>140 $\mathrm{mmHg}$ ) to transdermal GTN patch (5 $\mathrm{mg}$ ) or no GTN.

- The primary outcome was functional outcome (modified Rankin Scale, mRS) at day 90.

- Ipsilateral carotid stenosis was split: $<30 \%$; $30-<50 \% ; 50-<70 \%$; $\geq 70 \%$.

- Bilateral carotid stenosis was split: $<30 \% ; 30-$ $<50 \%$; $\geq 50 \%$.

Data are odds ratios (OR) with 95\% confidence intervals (CI) adjusted for baseline prognostic factors.

\section{RESULTS}

$2023(60.5 \%)$ ischaemic stroke participants had carotid imaging:

\begin{tabular}{clll} 
Ipsilateral: & \multicolumn{1}{c}{$\mathbf{n}$} & GTN & No GTN \\
$\cdot \quad<30 \%$ & 1431 & 726 & 705 \\
$\cdot \quad 30-<50 \%$ & 224 & 102 & 122 \\
$\cdot \quad 50-<70 \%$ & 148 & 77 & 71 \\
$\cdot \quad \geq 70 \%$ & 213 & 94 & 119
\end{tabular}

Bilateral:

$\begin{array}{llll}\text { - } \quad<30 \% & 1287 & 650 & 637 \\ \text { - } 30-<50 \% & 115 & 52 & 63 \\ \text { - } \geq 50 \% & 97 & 53 & 44\end{array}$

Compared with participants with $<30 \%, \geq 70 \%$ ipsilateral stenosis was associated with an unfavourable shift in mRS at 90 days (OR 1.88, 95\% CI 1.44-2.44, $\mathrm{p}<0.001$ ).

\section{RESULTS (Continued)}

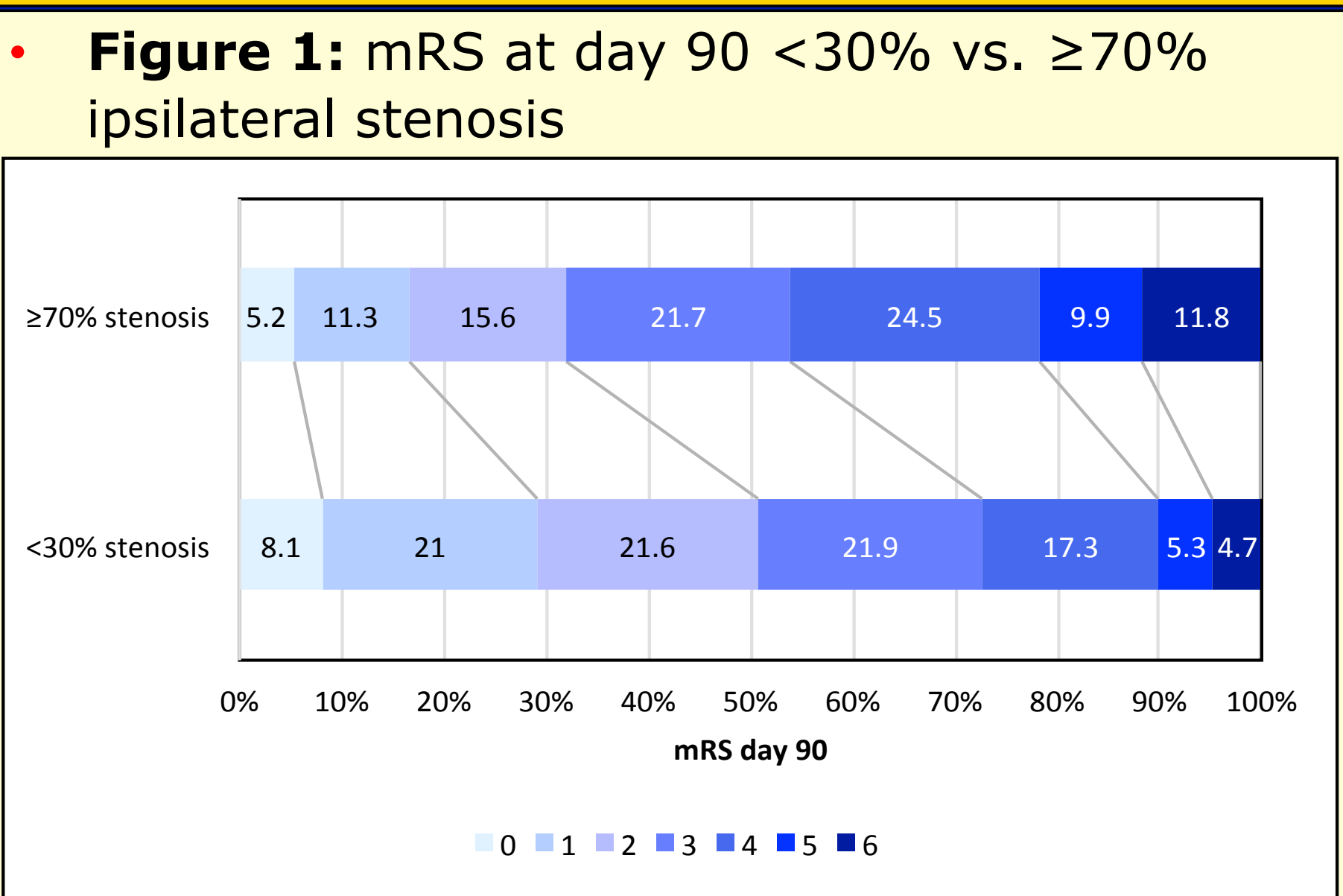

Figure 2: $\mathrm{mRS}$ at day 90 in those with $\geq 70 \%$ ipsilateral stenosis GTN vs. no GTN

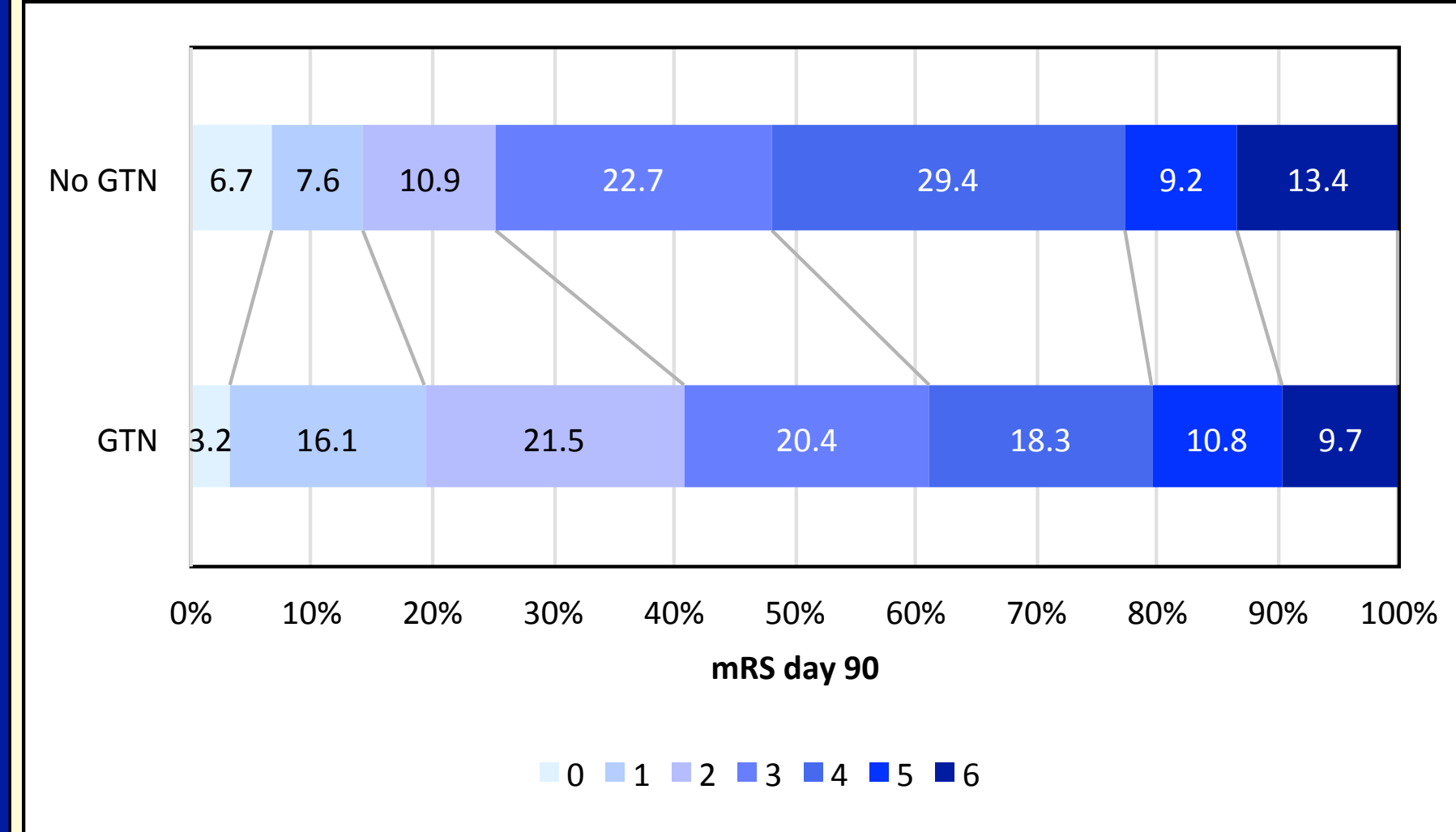

- Those with $\geq 70 \%$ stenosis who received GTN had a favourable shift in mRS (OR 0.56, 95\% CI $0.34-0.93, p=0.024$ ) compared to those who received no GTN.

- Tendencies towards less dependency, albeit non-significant, were seen in $30-<50 \%$ and $50-$ $<70 \%$ groups.

No differences in mRS were seen across groups of bilateral stenosis or between those who received GTN vs. no GTN.

\section{CONCLUSIONS}

- Severe ipsilateral carotid stenosis is associated with poorer functional outcome at 90 days following ischaemic stroke.

- GTN appears safe in acute stroke patients with ipsilateral or bilateral carotid stenosis. 